\title{
Side-effects of resveratrol in HepG2 cells: Reduced pten and increased bcl-xl mRNA expression
}

\author{
MIN ZHENG ${ }^{1}$, RUIFU CHEN ${ }^{1}$, HONGYUAN ZHONG ${ }^{1}$, QIUYAN LIN $^{1}$, \\ XIAOQIN WANG ${ }^{1}$, ZHIWEI ZHAO ${ }^{1}$ and LINNA XIE ${ }^{2}$ \\ ${ }^{1}$ Department of Biochemistry and Molecular Biology, School of Basic Medical Sciences, Fujian Medical University, \\ Fuzhou 350108; ${ }^{2}$ Department of Bioengineering, Fujian Vocational College of Bioengineering, Fuzhou 350007, P.R. China
}

Received April 16, 2012; Accepted September 6, 2012

DOI: $10.3892 / \mathrm{mmr} .2012 .1077$

\begin{abstract}
Resveratrol is a natural compound present in red grapes and red wine, and is often consumed in the human diet. The chemopreventive and chemotherapeutic potential of resveratrol in hepatocellular carcinoma (HCC), one of the most common types of liver cancer, has received much attention in recent years. However, the side-effects of resveratrol stimulation are of concern due to the high doses administered to humans. This study investigated the effects of resveratrol on the mRNA expression of pten and bcl-xl in HepG2 cells using semi-quantitative and quantitative PCR. This study demonstrated that $200 \mu \mathrm{mol} / 1$ resveratrol stimulation for $12 \mathrm{~h}$ resulted in the inhibition of HepG2 proliferation, reduced pten and increased bcl-xl mRNA expression. The data suggest that (a) the anticancer mechanism of resveratrol does not involve the induction of pten and inhibition of bcl-xl expression and (b) resveratrol induces a cellular self-protection response, which underlies the cellular chemoresistance against resveratrol in HepG2 cells.
\end{abstract}

\section{Introduction}

Resveratrol is a natural phenol produced by several plants and is found in the skin of red grapes and thus red wine. The anticancer activity of resveratrol has been studied since 1997, when Jang et al reported that resveratrol application prevented skin cancer development in a mouse model $(1,2)$. A number of researchers have suggested that resveratrol administration prevents skin and colon cancer (3) in animal models with artificially induced cancer. Recently, the effect of resveratrol against hepatocellular carcinoma (HCC) has received much interest $(4,5)$.

Correspondence to: Mr Min Zheng, Department of Biochemistry and Molecular Biology, School of Basic Medical Sciences, Fujian Medical University, 1 Xueyuan Road, Shangjie Zhen, Fuzhou 350108, P.R. China

E-mail: mzheng@mail.fjmu.edu.cn

Key words: resveratrol, pten, bcl-x1, HepG2
Experiments in cell cultures in vitro imply a number of mechanisms in the pharmacological activity of resveratrol. The responses of HepG2 cells to resveratrol were reported to include the inhibition of CYP1A1 transcription (6), upregulation of the expression of Sirtl and forkhead box O1 (FOXO1) (7), increase of JNK and ERK-1/2 MAP kinase activity and perforin expression (8), inhibition of VEGF expression (9) and downregulation of cyclin D1 (10) and protein S (11). The HepG2 human HCC cell line is one of the most suitable in vitro model systems to study hepatocarcinogenesis and drug targeting. It has been demonstrated that high doses of resveratrol treatment suppress HepG2 proliferation and subsequenctly induce cell death $(12,13)$. These molecular mechanisms may underlie the resveratrol-induced apoptosis or self-protection response of the HepG2 cells.

However, the effects of resveratrol on the mRNA expression levels of pten and bcl-xl are unknown. In the current study, the role of resveratrol on the regulation of pten and bcl-xl mRNA expression in HepG2 cells was investigated, using semi-quantitative and quantitative PCR analysis. The current study demonstrated that resveratrol treatment in HepG2 cells resulted in pten mRNA reduction and bcl-xl mRNA increase.

\section{Materials and methods}

Materials. Dulbecco's modified Eagle's medium (DMEM), fetal bovine serum (FBS), TRIzol reagent and all primers were purchased from Invitrogen (Carlsbad, CA, USA). Resveratrol was purchased from Sigma (St. Louis, MO, USA). M-MLV TransScript Reverse Transcriptase and TransStart Green qPCR Supermix were obtained from TransGen (Beijing, China).

Cell culture. The HepG2 human hepatocellular carcinoma cell line was cultured in DMEM supplemented with $10 \%$ (v/v) FBS and maintained at $37^{\circ} \mathrm{C} / 5 \% \mathrm{CO}_{2}$. The study was approved by the ethics committee of Fujian Medical University, Fuzhou, China.

RNA extraction and reverse transcription. HepG2 cells were treated with resveratrol $(200 \mu \mathrm{mol} / \mathrm{l})$ for $12 \mathrm{~h}$ and total RNA was extracted using TRIzol reagent according to the manufacturer's instructions. Total RNA was reversed transcribed using 
Table I. Sequences of proteins used in PCR.

\begin{tabular}{ll}
\hline Gene (no.) & \multicolumn{1}{c}{ Primer sequences } \\
\hline GAPDH & F: 5'-TGATGACATCAAGAAGGTGGTGAAG-3' \\
& R: 5'-TCCTTGGAGGCCATGTGGGCCAT-3' \\
pten (1) & F : 5'-ACCAGGACCAGAGGAAACCT-3' \\
& R: 5'-GCTAGCCTCTGGATTTGACG-3' \\
pten (2) & F: 5'-AACTTGCAATCCTCAGTTTG-3' \\
& R: 5'-GCATCTTGTTCTGTTTGTGG-3' \\
bcl-xl (1) & F: 5'-GAATGACCACCTAGAGCCTT-3' \\
& R: 5'-CATTTCCGACTGAAGAGTGA-3' \\
bcl-xl (2) & F : 5'-ACAGCAGCAGTTTGGATG-3' \\
& R: 5'-GGATGTCAGGTCACTGAATG-3' \\
\hline
\end{tabular}

F, forward; R, reverse.

$\mathbf{A}$
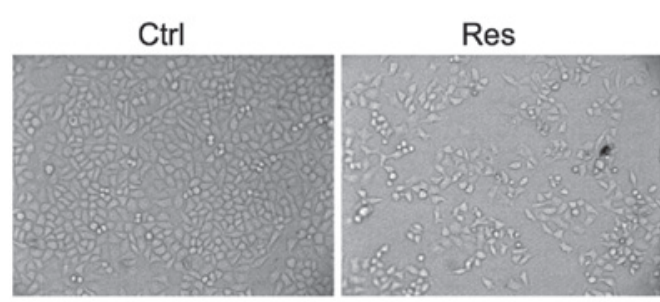

Figure 1. Effect of resveratrol on HepG2 cell proliferation, morphology and total RNA banding pattern. (A) HepG2 cells were stimulated with or without $200 \mathrm{mmol} / \mathrm{l}$ for $12 \mathrm{~h}$. Cell morphology and density were compared under microscopy with the same scale. (B) RNA extracted from both cells were compared. No clear RNA degradation was observed.

M-MLV TransScript Reverse Transcriptase according to the manufacturer's instructions.

Quantitative real-time PCR. Amplification and quantification of pten, bcl-xl and GAPDH genes were performed using TransStart Green qPCR Supermix according to the manufacturer's instructions. Real-time PCR primers for each gene were designed at ' $w w w . g e n e s c r i p t . c o m$ '. Two pairs of primers were designed for pten and bcl-xl. The forward and reverse primer sequences for each gene product are listed in Table I. Data were acquired with the Bio-Rad (Hercules, CA, USA) cfx96 real-time PCR detection system. For analysis, a standard curve was constructed for each gene in four serial 2-fold dilutions of cDNA stocks and then the amplification efficiency for each gene in each sample was determined. The amounts of mRNA expression for pten and bcl-xl were normalized to that of GAPDH, expressed as an arbitrary unit. The arbitrary unit for either pten or bcl-xl in the control was set as 1. Thus, the percentage of relative mRNA expression for either pten or bcl-xl in the sample was expressed as the arbitrary unit of the sample divided by that of the control.

Statistical analysis. Data are expressed as means \pm SEM. The two sample t-test was used to compare differences between the
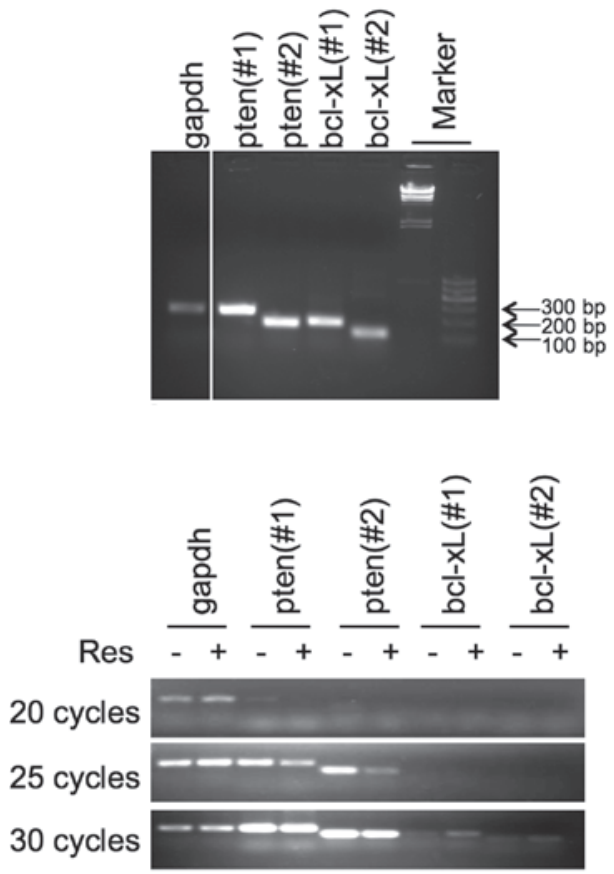

Figure 2. Semi-quantitative analysis of pten and bcl-xl mRNA expression in HepG2 cells stimulated as in Fig 1A. (A) The banding pattern of PCR products for each gene fragment. (B) Comparison of PCR products at 20, 25 and 30 cycles.
A

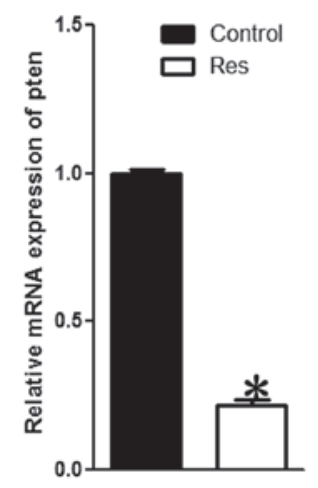

B

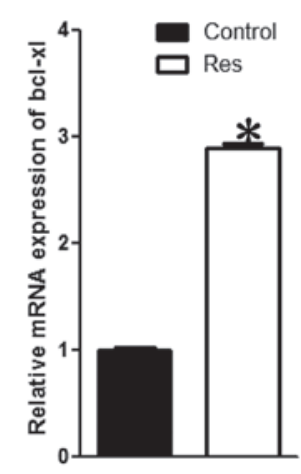

Figure 3. Quantitative analysis of pten and bcl-xl mRNA expression in HepG2 cells stimulated as in figure 1A. (A) Pten mRNA expression was determined. (B) Bcl-xl mRNA expression was determined. " $\mathrm{P}<0.05$ vs. control.

treated groups and their paired controls. P-values are indicated in the figures. Values of $\mathrm{P}<0.05$ were considered to indicate statistically significant differences.

\section{Results}

Suppression of resveratrol on HepG2 proliferation. HepG2 cells were seeded equally in 6-well plates and stimulated with $200 \mu \mathrm{mol} / 1$ resveratrol for various periods of time. Consistent with Yan et al (5), high doses of resveratrol lead to HepG2 apoptosis. The majority of the cells detached when treated with $200 \mu \mathrm{mol} / 1$ resveratrol for more than $24 \mathrm{~h}$. By contrast, the morphology of cells treated for $12 \mathrm{~h}$ was almost the same as that of the control, with the exception of cell density. As 
Fig. 1A shows, the density of stimulated HepG2 cells is approximately half of that of the control cells, supporting the hypothesis that resveratrol suppresses HepG2 cell proliferation. Cells were then harvested and RNAs were extracted. The RNAs were intact in control and treated cells (Fig. 1B).

Semi-quantitative analysis shows reduced pten and increased bcl-xl mRNA expression. Pten and bcl-xl are genes that regulate cell growth and cell death, respectively. The effect of resveratrol on mRNA expression of these genes was examined. RNAs were reversed transcribed into cDNA, which was subjected to semi-quantitative analysis. PCR products for each gene fragment from the cDNA are shown in Fig. 2A. PCR products were compared at 20, 25 and 30 cycles. As Fig. 2B shows, resveratrol exerted various effects on mRNA expression of pten and bcl-xl. Compared with GAPDH mRNA expression, pten mRNA expression was reduced, while bcl-xl mRNA expression was increased. The data were consistent between the two pairs of primers (numbers 1 and 2) for either pten or bcl-xl.

Quantitative analysis determines the fold of pten $m R N A$ reduction and bcl-xl mRNA increment. Quantitative PCR (qPCR) was performed to determine the fold of mRNA expression alteration. The cDNA samples were subjected to qPCR. As Fig. 3 shows, consistent with the semi-quantitative analysis, qPCR data demonstrates a reduction of pten mRNA and an increase of bcl-xl mRNA. The reduction fold for pten mRNA was determined to be $\sim 4.59$ (Fig. 3A), while the increment fold for bcl-xl mRNA was determined to be almost 2.90 (Fig. 3B), which were statistically significant.

\section{Discussion}

The carcinogenesis and chemotherapy resistance of cells in the majority of carcinomas are characterized by uncontrollable cell growth and the inhibition of cell death. Pten and bcl-xl are key protein factors in two pivotal pathways that control cell growth and death, respectively. The pten gene is one of the most commonly lost tumor suppressors in human cancer (14). Pten preferentially dephosphorylates phosphoinositide substrates such as PIP3, thereby negatively regulating the Akt/PKB-mTORC1 pathway, which is a pivotal signal contributing to cell growth and proliferation (15). Mutations or deletions of pten may both lead to increased cell proliferation and reduced cell death $(16,17)$. Frequent genetic inactivation of pten occurs in prostate and endometrial cancer and reduced expression is reported in numerous other tumor types (18). However, a number of tumors evade death signals by expressing anti-apoptotic proteins, including the pro-survival Bcl-2 family member, bcl-xl. Bcl-xl contains four bcl-2 homology $(\mathrm{BH})$ domains, which mediate its binding to Apaf-1 and subsequently the inhibition of apoptosis (19). Bcl-xl expression is enriched in prostate carcinoma (20), bladder cancer (21) and HCC (22). The downregulation of pten or overexpression of bcl-xl has been reported to play a role in the chemotherapy resistance in a variety of types of cancer.

In the current study, the effect of resveratrol on pten and bcl-xl mRNA expression in HepG2 cells was examined. Data revealed that resveratrol dynamically downregulated pten mRNA expression and upregulated bcl-xl mRNA expression, theoretically allowing the cells to survive. Therefore, the data suggest that resveratrol does not exert its antitumor activity through the inhibition of bcl-xl and induction of pten, and that resveratrol activates a cellular protection system against cell death, which underlies the chemoresistance to resveratrol in HepG2 cells.

Resveratrol is currently produced by chemical or biotechnological synthesis $(23,24)$ and is sold as a nutritional supplement or cancer chemopreventive agent. However, the in vivo effectiveness of resveratrol as a chemotherapy reagent is largely limited by its poor systemic bioavailability (25-27). Thus, high doses of resveratrol have been recommended in several clinical trials investigating its effects on colon cancer and melanoma $(28,29)$. Moreover, resveratrol has been reported to accumulate in the liver (30). These data present new concerns on the side-effects of resveratrol administration, which may break the balance of pro- and anti-growth signals in hepatocytes.

\section{Acknowledgements}

This study was supported by the National Natural Science Foundation of China (Grant No. 31100995) and the Natural Science Foundation of Fujian Province (Grant No. 2011J01187).

\section{References}

1. Jang M, Cai L, Udeani GO, et al: Cancer chemopreventive activity of resveratrol, a natural product derived from grapes. Science 275: 218-220, 1997.

2. Baur JA and Sinclair DA: Therapeutic potential of resveratrol: the in vivo evidence. Nat Rev Drug Discov 5: 493-506, 2006.

3. Santandreu FM, Valle A, Oliver J and Roca P: Resveratrol potentiates the cytotoxic oxidative stress induced by chemotherapy in human colon cancer cells. Cell Physiol Biochem 28: 219-228, 2011.

4. Weng CJ, Wu CF, Huang HW, Wu CH, Ho CT and Yen GC: Evaluation of anti-invasion effect of resveratrol and related methoxy analogues on human hepatocarcinoma cells. J Agric Food Chem 58: 2886-2894, 2010.

5. Yan F, Tian XM and Ma XD: Effects of resveratrol on growth inhibition and gap-junctional intercellular communication of HepG2 cells. Nan Fang Yi Ke Da Xue Xue Bao 26: 963-966, 2006 (In Chinese).

6. Ciolino HP, Daschner PJ and Yeh GC: Resveratrol inhibits transcription of CYP1A1 in vitro by preventing activation of the aryl hydrocarbon receptor. Cancer Res 58: 5707-5712, 1998.

7. Wang GL, Fu YC, Xu WC, Feng YQ, Fang SR and Zhou XH: Resveratrol inhibits the expression of SREBP1 in cell model of steatosis via Sirt1-FOXO1 signaling pathway. Biochem Biophys Res Commun 380: 644-649, 2009.

8. Lu CC and Chen JK: Resveratrol enhances perforin expression and NK cell cytotoxicity through NKG2D-dependent pathways. J Cell Physiol 223: 343-351, 2010.

9. Yu HB, Zhang HF, Zhang X, et al: Resveratrol inhibits VEGF expression of human hepatocellular carcinoma cells through a NF- $\mathrm{B}$-mediated mechanism. Hepatogastroenterology 57: 1241-1246, 2010

10. Parekh P, Motiwale L, Naik N and Rao KV: Downregulation of cyclin D1 is associated with decreased levels of p38 MAP kinases, Akt/PKB and Pak1 during chemopreventive effects of resveratrol in liver cancer cells. Exp Toxicol Pathol 63: 167-173, 2011.

11. Hiroto Y, Tadokoro K, Tsuda T, et al: Resveratrol, a phytoestrogen found in red wine, down-regulates protein $\mathrm{S}$ expression in HepG2 cells. Thromb Res 127: e1-7, 2011.

12. Ma XD, Yan F, Ma AD and Wang HJ: Resveratrol induces HepG2 cell apoptosis by depolarizing mitochondrial membrane. Nan Fang Yi Ke Da Xue Xue Bao 26: 406-408, 413, 2006 (In Chinese).

13. Zhou R, Fukui M, Choi HJ and Zhu BT: Induction of a reversible, non-cytotoxic S-phase delay by resveratrol: implications for a mechanism of lifespan prolongation and cancer protection. Br J Pharmacol 158: 462-474, 2009. 
14. Steck PA, Pershouse MA, Jasser SA, et al: Identification of a candidate tumour suppressor gene, MMAC1, at chromosome $10 \mathrm{q} 23.3$ that is mutated in multiple advanced cancers. Nat Genet 15: 356-362, 1997.

15. Chappell WH, Steelman LS, Long JM, et al: Ras/Raf/MEK/ERK and PI3K/PTEN/Akt/mTOR inhibitors: rationale and importance to inhibiting these pathways in human health. Oncotarget 2: 135-164, 2011

16. Chu EC and Tarnawski AS: PTEN regulatory functions in tumor suppression and cell biology. Med Sci Monit 10: RA235-241, 2004.

17. Li J, Yen C, Liaw D, et al: PTEN, a putative protein tyrosine phosphatase gene mutated in human brain, breast and prostate cancer. Science 275: 1943-1947, 1997.

18. Carracedo A, Alimonti A and Pandolfi PP: PTEN level in tumor suppression: how much is too little? Cancer Res 71: 629-633, 2011.

19. Adams JM and Cory S: The Bcl-2 protein family: arbiters of cell survival. Science 281: 1322-1326, 1998.

20. Castilla C, Congregado B, Chinchon D, Torrubia FJ, Japon MA and Saez C: Bcl-xL is overexpressed in hormone-resistant prostate cancer and promotes survival of LNCaP cells via interaction with proapoptotic Bak. Endocrinology 147: 4960-4967, 2006.

21. Hameed DA, Abdel Raheem AM, Mosad E, Hammouda HM, Kamel NA and Abdel Aziz MA: Bcl-XL and Bcl-2 expression in bilharzial squamous cell carcinoma of the urinary bladder: which protein is prognostic? Urology 72: 374-378, 2008.

22. Watanabe J, Kushihata F, Honda K, et al: Prognostic significance of Bcl-xL in human hepatocellular carcinoma. Surgery 135 604-612, 2004.
23. Farina A, Ferranti C and Marra C: An improved synthesis of resveratrol. Nat Prod Res 20: 247-252, 2006.

24. Trantas E, Panopoulos N and Ververidis F: Metabolic engineering of the complete pathway leading to heterologous biosynthesis of various flavonoids and stilbenoids in Saccharomyces cerevisiae. Metab Eng 11: 355-366, 2009.

25. Athar M, Back JH, Tang X, et al: Resveratrol: a review of preclinical studies for human cancer prevention. Toxicol Appl Pharmacol 224: 274-283, 2007.

26. Niles RM, Cook CP, Meadows GG, Fu YM, McLaughlin JL and Rankin GO: Resveratrol is rapidly metabolized in athymic (nu/nu) mice and does not inhibit human melanoma xenograft tumor growth. J Nutr 136: 2542-2546, 2006.

27. Wenzel E, Soldo T, Erbersdobler H and Somoza V: Bioactivity and metabolism of trans-resveratrol orally administered to Wistar rats. Mol Nutr Food Res 49: 482-494, 2005.

28. Nguyen AV, Martinez M, Stamos MJ, et al: Results of a phase I pilot clinical trial examining the effect of plant-derived resveratrol and grape powder on Wnt pathway target gene expression in colonic mucosa and colon cancer. Cancer Manag Res 1: 25-37, 2009.

29. Boocock DJ, Faust GE, Patel KR, et al: Phase I dose escalation pharmacokinetic study in healthy volunteers of resveratrol, a potential cancer chemopreventive agent. Cancer Epidemiol Biomarkers Prev 16: 1246-1252, 2007.

30. Notas G, Nifli AP, Kampa M, Vercauteren J, Kouroumalis E and Castanas E: Resveratrol exerts its antiproliferative effect on HepG2 hepatocellular carcinoma cells, by inducing cell cycle arrest and NOS activation. Biochim Biophys Acta 1760: 1657-1666, 2006. 